\title{
HEALTH RELATED OUTCOMES OF SUCCESSFUL DEVELOPMENT
}

\author{
Vladimír Kebza', Iva Šolcová2, Miloslav Kodl ${ }^{3}$, Věra Kernová ${ }^{3}$ \\ ${ }^{1}$ Department of Psychology, Czech University of Life Sciences, Prague, Czech Republic \\ ${ }^{2}$ Institute of Psychology, Czech Academy of Sciences, Prague, Czech Republic \\ ${ }^{3}$ National Institute of Public Health, Prague, Czech Republic
}

\section{SUMMARY}

Aim: Our longitudinal study of successful development would not be complete without an analysis of the health-related aspects of this successful development.

Method: A sample of 88 Czech adults (49 women, 39 men), participants of the Czech Longitudinal Study from 1956, was examined using a set of psychological methods and somato-physiological measures (weight, height, BMI, WHR, systolic and diastolic blood pressure), selected biochemical parameters (total cholesterol, triglycerides, glycaemia), a health questionnaire and measures focused on selected behavioural aspects of health (subjectively perceived health, overview of health problems and diseases, treatment).

Results: The values of obtained results both of correlational and regression analyses are mostly low, reflecting the variables studied.

Conclusion: The above mentioned data will offer a more comprehensive understanding of relationships and determinants between health, behavioural, psychological and social variables, predictors and outcomes within the adaptive functioning of personality during the life course.

Key words: successful development, longitudinal study, health related outcomes, selected psychological, somato-physiological and biochemical measures

Address for correspondence: V. Kebza, Czech University of Life Sciences, Department of Psychology, Kamýcká 129, 16521 Praha 6, Czech Republic. E-mail: kebza@pef.czu.cz

http://dx.doi.org/10.21101/cejph.a4232

\section{INTRODUCTION}

Our study of successful development would not be complete without an analysis of the health related aspects of successful development as such. The project is targeted at exploring the paths to successful development using empirical data from longitudinal studies of individuals since 1956 (Prague) and 1961 (Brno). We consider the state of present well-being as a manifestation of successfully mastered development. The variables expressing different aspects of well-being (life satisfaction, sense of coherence, life meaningfulness) have been monitored in the long term within these studies, and further constructs are included from this stage of the study. We will focus on resilience, human strengths and the strategies of selection, optimization and compensation as conceived by P. Baltes.

The developmental psychology of adults has developed as a fully fledged psychological discipline $(1,2)$ over the past twenty-five years. A canonical (normative) course of adult life (with consideration of gender) has been described, with respect to changes in status, roles, goals and tasks $(3,4)$. Broader theoretical methodological frameworks (often on an interdisciplinary basis) have been created for the purposes of research in human development. Of these, the theory of life course $(5,6)$, lifespan psychology (7), holistic interactionism (8) or developmental systems theory $(9,10)$ are of primary revelance.
A significant feature of the newly formulated theories is their departure from the study of individual stages of development and their psychological characteristics towards the study of general principles of lifespan development (e.g. processes of selection, optimization and compensation in P. Baltes' theoretical system). At the present time we observe another shift in focus, i.e. towards the subject of optimal development of the human, where developmental psychology meets personality psychology, clinical psychology, health psychology and the psychology of well-being in the common interest. Along with the concept of optimal development we are faced with other concepts such as successful development (7, $11,12)$, optimal ageing $(13)$ or healthy ageing $(14,15)$.

We can state that specialists in different fields of psychology, progressing through different ways, have come to be interested in the same subject, that of a happily or at least satisfactorily spent life. Of the theoretical fields of study it was mainly humanistic psychology which historically contributed to the study of optimal development, giving great attention to questions concerned with a fully/optimally developed personality ("fully functioning person") and living existentially (living a "good life"), and which also emphasized that the "good life" is a process, not a state of being $(16,17)$.

Of the newer psychological disciplines, it is primarily positive psychology which provides a theoretical anchor for the study of optimal development of the human (and among other sources significantly draws on humanistic psychology), and pursues an understanding of the positive forces in humans and their development (18). 


\begin{tabular}{|c|c|c|c|c|c|c|c|c|c|c|c|c|c|c|c|c|c|c|c|c|c|c|c|c|c|c|c|c|c|c|c|c|c|c|}
\hline 产 & & & & & & & & & & & & & & & & & & & & & & & & & & & & & & & & & & 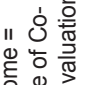 \\
\hline 串 & & & & & & & & & & & & & & & & & & & & & & & & & & & & & & & & $\stackrel{8}{-}$ & & 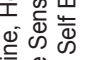 \\
\hline ֻั & & & & & & & & & & & & & & & & & & & & & & & & & & & & & & & $\stackrel{8}{-}$ & $\frac{\infty}{0}$ & & 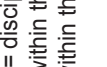 \\
\hline 흥 & & & & & & & & & & & & & & & & & & & & & & & & & & & & & & $\stackrel{8}{-}$ & 辛 & 8 & & 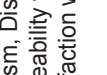 \\
\hline 总 & & & & & & & & & & & & & & & & & & & & & & & & & & & & & $\stackrel{8}{-}$ & $\begin{array}{l}\text { to } \\
\text { i }\end{array}$ & 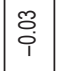 & ז்̇ & & 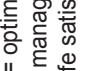 \\
\hline 今 & & & & & & & & & & & & & & & & & & & & & & & & & & & & $\stackrel{8}{-}$ & $\stackrel{\circ}{\circ}$ & $\begin{array}{l}8 \\
0 \\
i\end{array}$ & \begin{tabular}{|l|}
0 \\
0 \\
$i$ \\
$i$
\end{tabular} & $\stackrel{\frac{m}{0}}{0}$ & & $\begin{array}{ll}11 \\
\frac{11}{10} \\
1\end{array}$ \\
\hline 爰 & & & & & & & & & & & & & & & & & & & & & & & & & & & 8 & $\stackrel{\infty}{0}$ & 帝 & $\stackrel{ \pm}{*}$ & 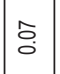 & $\mid$ & & \\
\hline$\overline{\bar{y}}$ & & & & & & & & & & & & & & & & & & & & & & & & & & 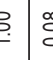 & : & $\underset{\substack{\infty \\
0 \\
0}}{ }$ & g & $\begin{array}{l}\widetilde{o} \\
\text { î }\end{array}$ & $\begin{array}{l}o \\
0 \\
0\end{array}$ & శ్ర్ర & & \\
\hline 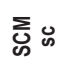 & & & & & & & & & & & & & & & & & & & & & & & & $\stackrel{8}{-}$ & 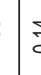 & $\begin{array}{l} \pm \\
5\end{array}$ & 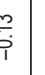 & ฐ & $\stackrel{9}{0}$ & 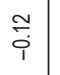 & 总 & $\mid \begin{array}{l} \\
\bar{c} \\
\bar{i}\end{array}$ & & "1" \\
\hline 해요 $\underline{x}$ & & & & & & & & & & & & & & & & & & & & & & & 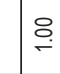 & $\exists$ & है & $\begin{array}{l}B \\
B\end{array}$ & 5 & $\bar{s}$ & ֶֻ. & $\stackrel{m}{\stackrel{m}{i}}$ & 8 & $\stackrel{\circ}{\circ}$ & & \\
\hline$\sum_{\infty}^{\Sigma} \bar{~}$ & & & & & & & & & & & & & & & & & & & & & & $\stackrel{8}{\square}$ & $\because$ & 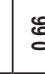 & $\dot{s}$ & $\begin{array}{c}\text { s. } \\
s\end{array}$ & $\frac{2}{i}$ & $\stackrel{\infty}{0}$ & $\overline{\tilde{o}}$ & \%̊: & \begin{tabular}{|l|}
\multirow{2}{*}{} \\
$\stackrel{p}{i}$
\end{tabular} & $\approx$ & & 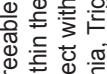 \\
\hline 啹 흐 & & & & & & & & & & & & & & & & & & & & & $\stackrel{8}{-}$ & $\stackrel{\alpha}{\alpha}$ & $\begin{array}{l}: \\
\vdots\end{array}$ & 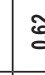 & $\vec{s}$ & 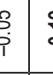 & : & $\frac{9}{0}$ & $\bar{s}$ & $\begin{array}{l}\text { to } \\
\text { i }\end{array}$ & $\begin{array}{l}8 \\
\\
i \\
\end{array}$ & $\begin{array}{l} \\
0 \\
0\end{array}$ & $\begin{array}{l}0 \\
0 \\
i \\
\end{array}$ & \\
\hline 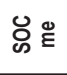 & & & & & & & & & & & & & & & & & & & & $\stackrel{8}{\circ}$ & : & $\hat{s}$ & $\stackrel{\leftrightarrow}{\circ}$ & $\stackrel{y}{9}$ & 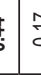 & $\begin{array}{c}n \\
i \\
i\end{array}$ & 总 & $\stackrel{0}{0}$ & : & 8 & $\mid \begin{array}{l}\hat{0} \\
\hat{i} \\
\end{array}$ & 音 & & \\
\hline $\mathscr{D}_{\infty}$ & & & & & & & & & & & & & & & & & & & $\stackrel{8}{\square}$ & 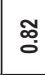 & 㚐 & $\begin{array}{l}\alpha \\
\alpha \\
c\end{array}$ & $\begin{array}{l} \\
\end{array}$ & s & $\dot{\xi}$ & ça & $\begin{array}{c}\text { ț } \\
\text { it }\end{array}$ & §̊ & ז్ర & 8 & $\stackrel{m}{i}$ & \begin{tabular}{|l|} 
\\
0 \\
0 \\
0
\end{tabular} & ס̊. & \\
\hline ه্లু & & & & & & & & & & & & & & & & & & $\stackrel{\leftrightarrow}{-}$ & $\stackrel{\infty}{0}$ & 8 & 홍 & $\hat{n}$ & 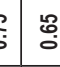 & 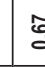 & $\grave{s}$ & $\begin{array}{l}3 \\
s\end{array}$ & $\begin{array}{c}v_{i}^{*} \\
\end{array}$ & $\overline{\check{\delta}}$ & ส & $\underset{\dot{Q}}{E}$ & 㝘 & $\%$ & 8 & \\
\hline dُّ & & & & & & & & & & & & & & & & & $\stackrel{8}{\circ}$ & $\stackrel{5}{0}$ & $\stackrel{9}{0}$ & $\stackrel{9}{0}$ & : & ã & $\frac{0}{0}$ & $\overline{\tilde{s}}$ & $\vdots$ & 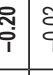 & î & $\stackrel{\infty}{\circ}$ & $\begin{array}{l}\text { d. } \\
\text { d. }\end{array}$ & 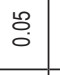 & 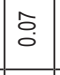 & \begin{tabular}{|l|l|l|}
0 \\
0
\end{tabular} & : & \\
\hline ڤั̀ & & & & & & & & & & & & & & & & 3 & 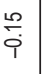 & $\begin{array}{l}\infty \\
\stackrel{i}{i} \\
\end{array}$ & $\begin{array}{c}0 \\
0 \\
i \\
i\end{array}$ & $\hat{i}$ & \begin{tabular}{|c}
$\overline{0}$ \\
$\bar{i}$
\end{tabular} & a & 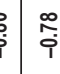 & 舟 & 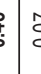 & Ș & s: & $\stackrel{\infty}{i}$ & $\overline{\bar{i}}$ & $\approx$ & 号 & $\frac{\text { 임 }}{\grave{1}}$ & 嵩 & \\
\hline$\frac{x}{4}$ & & & & & & & & & & & & & & 8 & & \begin{tabular}{c|c}
0 \\
0 \\
0
\end{tabular} & $\frac{9}{9}$ & 胥 & $\begin{array}{l}0 \\
0 \\
i \\
i\end{array}$ & $\stackrel{\bar{i}}{i}$ & $\begin{array}{l}2 \\
0 \\
i\end{array}$ & $\begin{array}{l}9 \\
\vdots \\
9\end{array}$ & $\begin{array}{ll} \\
\\
\end{array}$ & 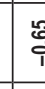 & 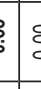 & $\begin{array}{l}P \\
S\end{array}$ & 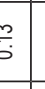 & శ్ & $\begin{array}{l}\text { ș } \\
\mathbf{i} \\
\end{array}$ & \begin{tabular}{|c|} 
\\
\\
0
\end{tabular} & $\stackrel{\circ}{\circ}$ & \begin{tabular}{|c|} 
\\
$\bar{i}$ \\
\end{tabular} & 8 & \\
\hline 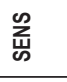 & & & & & & & & & & & & & $\stackrel{8}{-}$ & 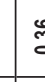 & 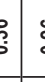 & 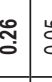 & : & ্ָণ & $\begin{array}{c}\tilde{m} \\
\tilde{i} \\
\end{array}$ & $\underset{i}{i}$ & 兽 & $\bar{c}$ & 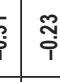 & 年 & 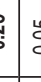 & ?: & s. & $\stackrel{ \pm}{ \pm}$ & $\stackrel{8}{\circ}$ & 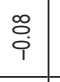 & $\cong$ & \begin{tabular}{|l|} 
\\
0 \\
0 \\
\end{tabular} & ্ָডি. & \\
\hline 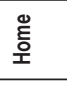 & & & & & & & & & & & & $\stackrel{8}{\circ}$ & $\stackrel{\circ}{\circ}$ & శ̊ & 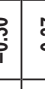 & 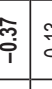 & $\stackrel{\frac{m}{0}}{\circ}$ & : & 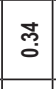 & న్ & 梁 & $\stackrel{a}{a}$ & $\stackrel{3}{s}$ & §̊ & b & 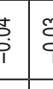 & 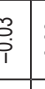 & $\begin{array}{l}8 \\
0 \\
0\end{array}$ & $\begin{array}{l}8 \\
\\
\end{array}$ & $\begin{array}{l} \pm \\
0 \\
i \\
\end{array}$ & $\begin{array}{l}0 \\
0 \\
\end{array}$ & $\begin{array}{l}8 \\
0 \\
i \\
\end{array}$ & 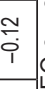 & \\
\hline$\stackrel{\mathscr{D}}{\Delta}$ & & & & & & & & & & & $\stackrel{8}{\circ}$ & స̃ & $\bar{\partial}$ & जे & 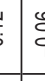 & 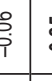 & : & $\stackrel{\circ}{\circ}$ & 兽 & 总 & : & $\bar{c}$ & $\stackrel{ \pm}{0}$ & $\frac{\infty}{c}$ & : & $\frac{1}{i}$ & s. & 8 & ${ }_{i}^{E}$ & $\stackrel{\vec{c}}{0}$ & $\stackrel{m}{0}$ & \begin{tabular}{|l|}
$\infty$ \\
0 \\
$i$ \\
\end{tabular} & . & \\
\hline 홍 & & & & & & & & & & $\stackrel{8}{\longrightarrow}$ & @o. & 晜 & 㐁 & 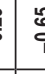 & 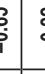 & 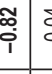 & 辛 & $\bar{\sigma}_{0}$ & $\begin{array}{l}\mathbb{N} \\
0 \\
0\end{array}$ & ? & $\stackrel{\Re}{0}$ & $\hat{c}$ & $\begin{array}{l}\dot{0} \\
\dot{0}\end{array}$ & g & $\dot{c}$ & s. & t: & : & ฐ & $\tilde{\Xi}$ & $\bar{\delta}$ & $\begin{array}{l}8 \\
0 \\
\end{array}$ & $\begin{array}{l}8 \\
0 \\
0\end{array}$ & \\
\hline 总。 & & & & & & & & & $\stackrel{8}{-}$ & $\stackrel{2}{\circ}$ & శ్ & $\stackrel{\infty}{0}$ & 离 & ç & 蜶 & : & 范 & 是 & 急 & $\overline{\text { sog }}$ & 胞 & \& & 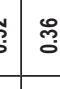 & $\stackrel{\infty}{c}$ & $\frac{1}{9}$ & 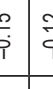 & 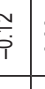 & : & $\tilde{\check{c}}$ & $\begin{array}{l}\stackrel{0}{0} \\
c \\
c\end{array}$ & 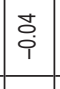 & $\begin{array}{l}0 \\
0 \\
i \\
i\end{array}$ & 范 & 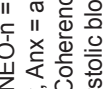 \\
\hline 石 & & & & & & & & $\stackrel{8}{\circ}$ & 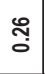 & \&̊ & 总 & $\stackrel{\text { \& }}{0}$ & $\stackrel{8}{\circ}$ & $\vec{i}$ & & $\begin{array}{c}m_{i}^{\infty} \\
i\end{array}$ & $\stackrel{\cong}{0}$ & $\stackrel{m}{0}$ & $\bar{s}$ & $\stackrel{\text { \& }}{\circ}$ & I & a & S. & $\frac{\infty}{a}$ & $\begin{array}{l}0 \\
\vdots\end{array}$ & 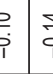 & 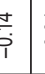 & \begin{tabular}{l|} 
to \\
i
\end{tabular} & 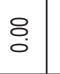 & à & 8 & 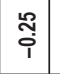 & : & \\
\hline 물。 & & & & & & $£$ & $\stackrel{\xi}{\xi}$ & : & $\stackrel{m}{0}$ & న్రి & 吕 & o̊ & ָ̃ & $\begin{array}{l}8 \\
9 \\
9\end{array}$ & s & $\frac{1}{i}$ & 范 & 范 & $\stackrel{2}{0}$ & జ్ & $\stackrel{\circ}{\circ}$ & : & io & $\stackrel{n}{i}$ & 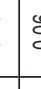 & 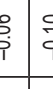 & $\frac{2}{i}$ & $\stackrel{5}{i}$ & $\begin{array}{l}\text { ț } \\
\dot{i}\end{array}$ & $\stackrel{\infty}{0}$ & $\tilde{O}$ & $\begin{array}{l}\tilde{0} \\
\bar{i} \\
\end{array}$ & $\frac{0}{\circ}$ & \\
\hline 层。 & & & & & $\stackrel{8}{-}$ & $\xi$ & הָ & ฐ্ & 莒 & : & Б্口. & $\stackrel{5}{0}$ & 岕 & 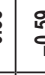 & 㸃 & $\begin{array}{l}8 \\
\\
i\end{array}$ & $\stackrel{t}{ \pm}$ & f & $\dddot{8}$ & 总 & $\overline{0}$ & 8 & : & लू & 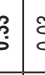 & s. & 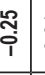 & 总 & $\stackrel{0}{\circ}$ & 容 & 容 & \begin{tabular}{l|}
8 \\
0 \\
0
\end{tabular} & $\tilde{\Xi}$ & \\
\hline 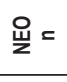 & & & & 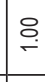 & $\begin{array}{l}5 \\
0 \\
9\end{array}$ & 䔬 & $\bar{i}^{\prime}$ & 足 & $\begin{array}{l}\text { ? } \\
i \\
i\end{array}$ & $\underset{i}{\mathbb{N}}$ & 웅 & ঙ্ণి & 尔 & 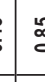 & 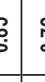 & 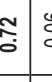 & $\begin{array}{l}8 \\
i \\
i \\
\end{array}$ & $\begin{array}{c}: \\
i \\
\end{array}$ & 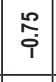 & $\begin{array}{l}\circ \\
\\
\end{array}$ & $\begin{array}{l}2 \\
i \\
i\end{array}$ & A & $\begin{array}{l}: \\
\\
i\end{array}$ & $\begin{array}{l}\infty \\
0 \\
9\end{array}$ & $\dot{\xi}$ & 草 & 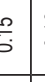 & $\stackrel{\circ}{i}$ & $\bar{i}$ & $\frac{9}{\circ}$ & $\begin{array}{l}0 \\
i \\
i \\
\end{array}$ & 8 & 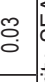 & \\
\hline 恿 & & & 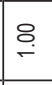 & 辛 & 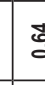 & 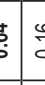 & 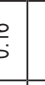 & I & : & ז & $\stackrel{m}{0}$ & : & 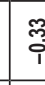 & 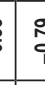 & 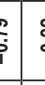 & 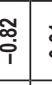 & $\bar{s}$ & $\tilde{O}$ & $\begin{array}{l}\hat{1} \\
0 \\
0\end{array}$ & R & 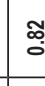 & $\stackrel{\infty}{a}$ & $\stackrel{0}{8}$ & 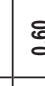 & $\grave{s}$ & : & 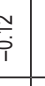 & ฐ్ర & శ్రి & $\begin{array}{l}\tilde{O} \\
\bar{i}\end{array}$ & $\begin{array}{l}0 \\
0 \\
i \\
i\end{array}$ & $\begin{array}{ll}0 \\
0 \\
0\end{array}$ & 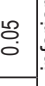 & \\
\hline$\underline{\underline{\underline{E}}}$ & & $\stackrel{8}{-}$ & \begin{tabular}{|c}
$\stackrel{8}{8}$ \\
$i$ \\
$i$
\end{tabular} & $\stackrel{\infty}{0}$ & : & is & $\begin{array}{c}0 \\
\vdots \\
i \\
\end{array}$ & 悉 & $\begin{array}{l}\text { F } \\
i \\
\end{array}$ & $\begin{array}{c}\text { co } \\
i \\
i\end{array}$ & $\begin{array}{l}8 \\
\text { i } \\
\end{array}$ & i. & \% & 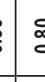 & 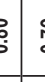 & $\begin{array}{l}0 \\
0 \\
0\end{array}$ & $\bar{i}$ & $\begin{array}{c}\tilde{i} \\
\hat{i} \\
\end{array}$ & \begin{tabular}{|l|} 
\\
0 \\
$i$ \\
\end{tabular} & $\begin{array}{l}\text { Ta } \\
\text { i }\end{array}$ & $\begin{array}{c}0 \\
0 \\
i \\
i\end{array}$ & $\begin{array}{c}\bar{\alpha} \\
c \\
c\end{array}$ & $\begin{array}{l}0 \\
\vdots \\
\vdots \\
i\end{array}$ & : & $\begin{array}{c}\dot{c} \\
\vdots \\
\vdots\end{array}$ & $\vec{i}$ & ? & 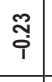 & న్ని & : & $\approx$ & \begin{tabular}{|l|} 
\\
0 \\
0 \\
\end{tabular} & : & \\
\hline 总 & $\stackrel{8}{-}$ & M. & 옹 & : & 品 & $\begin{array}{lll}8 \\
b\end{array}$ & $\frac{6}{5}$ & : & 䁶 & t5 & $\stackrel{0}{0}$ & $\stackrel{9}{0}$ & 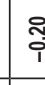 & 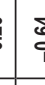 & 寞 & 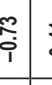 & f. & $\bar{\Xi}$ & $\because$ & : & ז. & $\tilde{\Sigma}$ & $\stackrel{5}{5}$ & $\bar{s}$ & 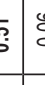 & s: & $\frac{1}{i}$ & $\frac{9}{5}$ & శ్త & $\bar{o}_{0}$ & $\begin{array}{l}\stackrel{\leftrightarrow}{0} \\
\dot{p}\end{array}$ & ฉి & 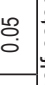 & \\
\hline & 氮 & $\underline{\underline{\underline{s}}}$ & 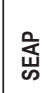 & 产 & 㝴 & $\begin{array}{l}\dot{v} \\
\vec{z}\end{array}$ & 兑 & 总 & 虽 & 흥 & 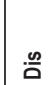 & 윻 & 恙 & z & $\frac{x}{1}$ & ‡ & 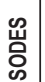 & 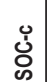 & 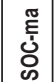 & 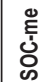 & 总 & 这 & 竞 & 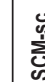 & 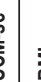 & 商 & 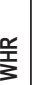 & $\stackrel{\infty}{\infty}$ & 호 & 흥 & $\frac{0}{\mathrm{O}}$ & 을 & & \\
\hline
\end{tabular}


The results of the latest examination so far undertaken within the Prague longitudinal study have been summarized in the final report on project GACR no. 406/04/0027 entitled "Psychological aspects of health behaviours in adulthood of persons followed-up from the birth" (19). The results show, among other things, that the Prague study has successfully progressed through the next stage and the following of the cohort and its active cooperation continues.

The aim of this study is to follow up the ongoing Brno and Prague longitudinal study of a cohort of the Czech population and obtain data for mapping the development of personality characteristics, levels of their mental resilience, state of health and development of the health-related complex of their behaviour. The project is based on an interdisciplinary approach and the analysis of relations between psychological and anthropological aspects and health variables, as developing over time.The principles underlying the above approach are based on both the research and clinical findings and the main programme documents of the World Health Organization (WHO). Within the gradual process of development of the global strategy aiming for health for all in the 21st Century(WHO/Euro,1998), significant relations have been identified step by step between all the dimensions of the holistically conceived health and the essential health risks, including the proposed strategies for increasing the level of health protection and promotion and enhancing effectiveness of illness prevention.

Significant attention is paid to the study of somatic-physiological, genetic, biochemical, psychological, and social impacts relating to risk factors of cardiovascular diseases (CVD), which since the second half of the 20th century have topped the list of the most frequen causes of mortality worldwide, while up to $80 \%$ of premature mortality from CVD are identified as preventable (WHO, 2009).

The basic methodology of the substudy consists of an updated questionnaire examination which formed the basis of the previous stages of the longitudinal study, now completed by an anthropological and basic internal medical examination involving the collection of a small amount of capillary blood for biochemical analyses, carried out by standard methodology using a Reflotron device which the National Institute of Public Health has at its disposal.

\section{MATERIALS AND METHODS}

Data were collected through individual examination in the National Institute of Public Health in Prague, participants received a compensation amounting to CZK 1,200.

The sample of 88 Czech adults (49 women, 39 men), participants of the Czech Longitudinal Study from 1956, was examined by the following set of psychological methods: NEO-FFI (20, 21), Rosenberg Self-Esteem Scale (22), General Self-Efficacy Scale (23), Rokeach Values Survey (24), Logo-Test (25), Sense of Coherence Scale (26), Life History Calendar (27), Values in Action Inventory of Strengths $(28,29,30)$, The Measure of Selection, Optimization, and Compensation (SOC) by Self-report (31), PPVT Vocabulary Scale (32), Nonword Repetition Task (33), a structured interview on life goals and medical examination. The above mentioned sample was also examined by selected somatophysiological measures (weight, height, BMI, WHR, systolic and diastolic blood pressure), selected biochemical parameters (total cholesterol, triglycerides, glycaemia) a health questionnaire and measures focused on selected behavioural aspects of health (subjectively perceived health, overview of health problems and diseases, treatment).

\section{RESULTS}

Correlational analysis revealed remarkable values (we present the three highest values) between self-esteem and self-appreciation, as well as between self-esteem and the total score and subscores of Sense of Coherence (SOC) (Total Score of the SOC Scale, Comprehesibility within the SOC Scale, Manageability within the SOC Scale, Meaningfulness within the SOC Scale) (Table 1). Self-esteem demonstrates a statistically significant correlation with self-appreciation, self-respect, and total score of SOC, and negative correlation with inferiority, depression, and anxiety. Inferiority revealed statistically significant values of correlation with depression, anxiety and neuroticism and significant values of negative correlation with self-appreciation, self-respect and the total score of SOC. Self-appreciation presented a statistically significant correlation with self-respect, and the total score and subscores of SOC, and negative correlation with depression, anxiety and neuroticism. Neuroticism showed a statistically significant correlation with anxiety, depression and interpersonal sensitivity, and negative correlation with self-respect, manageability within SOC and the total score of SOC. Extraversion presented a statistically significant correlation with self-respect, manageability and meaningfulness, and negative correlation with depression, anxiety, and interpersonal sensitivity. Openness to experience demonstrated a statistically significant correlation with meaningfulness within SOC and optimism, and negative correlation with interpersonal sensitivity. Agreeableness presented a statistically significant correlation with discipline, manageability within SOC, life satisfaction and negative correlation with depression and triglycerides. Conscientiousness expressed a statistically significant correlation with social desirability, total score of SOC, self-respect and negative correlation with anxiety and depression. Optimism presented a statistically significant correlation with self-respect, total score of SOC, and manageability within SOC, and negative correlation with depression, anxiety, and interpersonal sensitivity. Discipline demonstrated a statistically significant correlation with favourable memories of home, social desirability and self-concept, and negative correlation with smoking. Favourable memories of home showed a statistically significant correlation with life satisfaction, manageability within SOC, and total score of SOC, and negative correlation with depression and anxiety. Interpersonal sensitivity demonstrated a statistically significant correlation with anxiety and depression, and negative correlation with manageability within SOC, self-respect, and total score of SOC. Anxiety presented a statistically significant correlation with depression, and negative correlation with manageability within SOC, self-respect, and total score of SOC. Social desirability expressed a statistically significant correlation with self-concept, self-respect, and total score of SOC, and negative correlation with body-mass index (BMI). Comprehensibility within SOC presented a statistically significant correlation besides other subscores and total score of SOC with self-respect, self-concept, and life satisfaction. Manage- 
ability within SOC expressed a statistically significant correlation besides other subscores and total score of SOC with self-respect, life satisfaction, and self-concept. Meaningfulness within SOC demonstrated a significant correlation besides the total score of SOC with self-respect, life satisfaction, and self-concept. The total SOC score also presented a statistically significant correlation with self-respect, life satisfaction, and self-concept. Self-respect presented a statistically significant correlation with life satisfaction, self-concept, and diastolic blood pressure. Life satisfaction demonstrated a statistically significant correlation with self-concept, and both systolic and diastolic blood pressure. Also self-concept expressed a statistically significant correlation with both systolic and diastolic blood pressure. Regarding the somato-physiological and biochemical measures, the highest values of correlation were found out between life satisfaction and both systolic and diastolic blood pressure, between self-respect and diastolic blood pressure, and between self-appreciation and both systolic and diastolic blood pressure.

\section{Comparison of Data from 2004 and 2014}

As regards the physical examinations, we recorded statistically significant differences between both systolic and diastolic blood pressure, waist-hip ratio (WHR) and body mass index (BMI) (Table 2).

\section{Regression Analysis}

Regression analysis was used (Table 3 and 4). As regards predictors of Shirom-Melamed Burnout Measure (SMBM), we

Table 2. Physical examination - measured data, comparison of 2014 vs. 2004

\begin{tabular}{|l|c|c|c|c|c|}
\hline Variable & Observations & Mean & SE & SD & $95 \%$ Cl \\
\hline Body mass index 2014 & 78 & 27.01 & 0.58 & 5.09 & $25.86-28.16$ \\
\hline Body mass index 2004 & 78 & 25.59 & 0.51 & 4.55 & $24.57-26.62$ \\
\hline Waist-hip ratio 2014 & 73 & 0.974 & 0.014 & 0.124 & $0.945-1.002$ \\
\hline Waist-hip ratio 2004 & 73 & 0.882 & 0.010 & 0.085 & $0.862-0.902$ \\
\hline Systolic blood pressure 2014 & 69 & 130.45 & 2.11 & 17.51 & $126.24-134.66$ \\
\hline Systolic blood pressure 2004 & 69 & 125.75 & 1.50 & 12.42 & $122.77-128.74$ \\
\hline Diastolic blood pressure 2014 & 69 & 81.81 & 1.52 & 12.60 & $78.78-84.84$ \\
\hline Diastolic blood pressure 2004 & 69 & 79.42 & 1.09 & 9.06 & $77.24-81.60$ \\
\hline
\end{tabular}

Table 3. Regression analysis - predictors of Shirom-Melamed Burnout Measure (SMBM) (N=71)

\begin{tabular}{|c|c|c|c|}
\hline & $\begin{array}{c}\text { SMBM } \\
\text { physical fatique }\end{array}$ & $\begin{array}{c}\text { SMBM } \\
\text { cognitive weariness }\end{array}$ & $\begin{array}{c}\text { SMBM } \\
\text { emotional exhaustion }\end{array}$ \\
\hline Diabetes of respondent & -0.03 & -0.09 & -0.06 \\
\hline Tumors of respondent & -0.24 & $-0.38^{*}$ & $-0.37^{*}$ \\
\hline Asthma of respondent & 0.16 & -0.13 & -0.01 \\
\hline Cardiovascular diseases in family & 0.18 & 0.02 & 0.12 \\
\hline Tumors in family & 0.06 & -0.02 & 0.01 \\
\hline Diabetes in family & 0.00 & 0.09 & -0.15 \\
\hline Asthma in family & -0.16 & $-0.28^{*}$ & $-0.22^{*}$ \\
\hline Regular smoking & -0.08 & -0.17 & -0.21 \\
\hline Regular alcohol consumption & 0.11 & -0.13 & -0.11 \\
\hline Regular physical activity & $0.30^{*}$ & $0.21^{*}$ & 0.01 \\
\hline Health index (health problems during last 6 months) & 0.26 & $0.63^{*}$ & 0.60 \\
\hline Body mass index & 0.10 & 0.03 & 0.13 \\
\hline Waist-hip ratio & $-0.25^{*}$ & $-0.30^{*}$ & -0.11 \\
\hline Systolic blood pressure & $-0.51^{*}$ & -0.25 & $-0.32^{*}$ \\
\hline Diastolic blood pressure & $0.24^{*}$ & 0.05 & 0.03 \\
\hline Total cholesterol & $-0.29^{*}$ & $-0.25^{*}$ & $-0.25^{*}$ \\
\hline Glycaemia & -0.15 & -0.13 & $-0.32^{*}$ \\
\hline Triglycerides & -0.11 & -0.22 & -0.07 \\
\hline Health status (subjective evaluation of health status) & 0.16 & 0.04 & -0.14 \\
\hline Cardiovascular diseases & -0.24 & -0.50 & -0.53 \\
\hline
\end{tabular}

${ }^{*} 5 \%$ level of statistical significance 
Table 4. Regression analysis - predictors of Sense of Coherence (SOC)

\begin{tabular}{|c|c|c|c|c|}
\hline Variable & $\begin{array}{c}\mathrm{SOC} \\
\text { comprehesibility }\end{array}$ & $\begin{array}{c}\text { SOC } \\
\text { manageability }\end{array}$ & $\begin{array}{c}\text { SOC } \\
\text { meaningfulness }\end{array}$ & $\begin{array}{c}\text { SOC } \\
\text { total score }\end{array}$ \\
\hline Health index in 45 years of age & $-0.73^{*}$ & $-0.76^{*}$ & $-0.84^{*}$ & $-0.88^{*}$ \\
\hline Health index in 55 years of age & -0.44 & $-0.56^{*}$ & -0.53 & $-0.56^{*}$ \\
\hline Body mass index in 45 years of age & 0.10 & 0.06 & -0.08 & 0.04 \\
\hline Body mass index in 55 years of age & -0.07 & -0.11 & -0.14 & -0.12 \\
\hline Waist-hip ratio in 45 years of age & $0.21^{*}$ & 0.10 & 0.17 & 0.19 \\
\hline Waist-hip ratio in 55 years of age & $0.26^{*}$ & 0.17 & 0.17 & $0.23^{*}$ \\
\hline Systolic blood pressure in 45 years of age & $0.30^{*}$ & $0.43^{*}$ & $0.32^{*}$ & $0.40^{*}$ \\
\hline Systolic blood pressure in 55 years of age & $0.34^{*}$ & $0.42^{*}$ & $0.27^{*}$ & $0.38^{*}$ \\
\hline Diastolic blood pressure in 45 years of age & 0.05 & 0.15 & 0.19 & 0.15 \\
\hline Diastolic blood pressure in 55 years of age & 0.00 & 0.02 & -0.03 & 0.00 \\
\hline Total cholesterol in 45 years of age & $0.21^{*}$ & $0.26^{*}$ & 0.10 & $0.22^{*}$ \\
\hline Total cholesterol in 55 years of age & 0.18 & $0.28^{*}$ & 0.15 & $0.23^{*}$ \\
\hline Glycaemia in 45 years of age & -0.17 & -0.05 & 0.02 & -0.09 \\
\hline Glycaemia in 55 years of age & -0.11 & 0.02 & -0.01 & -0.04 \\
\hline Triglycerides in 45 years of age & $0.33^{*}$ & $0.40^{*}$ & $0.29^{*}$ & $0.39^{*}$ \\
\hline Triglycerides in 55 years of age & 0.20 & $0.26^{*}$ & $0.23^{*}$ & $0.25^{\star}$ \\
\hline Regular smoking in 45 years of age & 0.04 & 0.12 & $0.33^{*}$ & 0.17 \\
\hline Regular smoking in 55 years of age & 0.11 & 0.17 & 0.19 & 0.17 \\
\hline Regular alcohol consumption in 45 years of age & $0.19^{*}$ & 0.07 & 0.10 & 0.14 \\
\hline Regular alcohol consumption in 55 years of age & $0.19^{*}$ & 0.15 & 0.14 & $0.18^{*}$ \\
\hline Subjective evaluation of health in 45 years of age & -0.03 & 0.21 & 0.17 & 0.12 \\
\hline Subjective evaluation of health in 55 years of age & $-0.22^{*}$ & -0.03 & -0.10 & -0.13 \\
\hline Cardiovascular diseases in 45 years of age & $0.55^{*}$ & $0.43^{*}$ & $0.51^{*}$ & $0.57^{*}$ \\
\hline Cardiovascular diseases in 55 years of age & $0.44^{*}$ & $0.47^{*}$ & $0.35^{*}$ & $0.47^{*}$ \\
\hline Diabetes in 45 years of age & 0.19 & 0.03 & 0.14 & 0.14 \\
\hline Diabetes in 55 years of age & $0.25^{*}$ & 0.19 & $0.22^{*}$ & $0.24^{*}$ \\
\hline Tumors in 45 years of age & 0.21 & $0.24^{*}$ & $0.40^{*}$ & $0.32^{*}$ \\
\hline Tumors in 55 years of age & $0.23^{*}$ & 0.17 & $0.33^{*}$ & $0.26^{*}$ \\
\hline Asthma in 45 years of age & $0.24^{*}$ & 0.22 & 0.15 & $0.24^{*}$ \\
\hline Asthma in 55 years of age & $0.26^{*}$ & $0.25^{*}$ & 0.13 & $0.24^{*}$ \\
\hline Cardivascular diseases in family in 45 years of age & -0.08 & 0.03 & 0.08 & 0.01 \\
\hline Cardivascular diseases in family in 55 years of age & -0.05 & -0.07 & -0.06 & -0.07 \\
\hline Tumors in family in 45 years of age & -0.02 & 0.10 & 0.11 & 0.06 \\
\hline Tumors in family in 55 years of age & 0.06 & 0.10 & 0.03 & 0.07 \\
\hline Diabetes in family in 45 years of age & 0.11 & 0.19 & $0.26^{*}$ & $0.21^{*}$ \\
\hline Diabetes in family in 55 years of age & 0.03 & 0.08 & 0.03 & 0.05 \\
\hline Asthma in family in 45 years of age & -0.02 & $-0.21^{*}$ & -0.04 & -0.10 \\
\hline Asthma in family in 55 years of age & -0.13 & -0.16 & 0.04 & -0.10 \\
\hline
\end{tabular}

${ }^{*} 5 \%$ level of statistical significance

detected the following statistically significant predictors of physical fatigue: physical activity, diastolic blood pressure, and the following negative predictors: WHR, systolic blood pressure, and total cholesterol. Regarding predictors of SOC, we recorded the following statistically significant predictors of comprehensibility: health index at age 45 years of participants, WHR in both 45 and 55 years, systolic blood pressure in both 45 and 55 years, total cholesterol in 45 years, triglycerides in 45 years, alcohol consump- tion both in 45 and 55 years, cardiovascular disease both in 45 and 55 years, diabetes and tumors in 55 years, asthma in both 45 and 55 years, and as negative predictors health status in 45 years. As predictors of manageability within SOC we identified health index in both 45 and 55 years, systolic blood pressure in both 45 and 55 years, total cholesterol in both 45 and 55 years, triglycerides in both 45 and 55 years, cardiovascular disease in both 45 and 55 years, tumors in 45 years, asthma in 55 years, and asthma in family 
Table 5. Questionnaire - direct questions/over limit values

\begin{tabular}{|l|c|c|c|c|c|}
\hline Variable & Observations & Mean & SE & SD & $95 \%$ CI \\
\hline Smoking in 2014 & 77 & 0.260 & 0.050 & 0.441 & $0.160-0.360$ \\
\hline Smoking in 2004 & 77 & 0.260 & 0.050 & 0.441 & $0.160-0.360$ \\
\hline Regular alcohol consumption in 2014 & 77 & 0.195 & 0.045 & 0.399 & $0.104-0.285$ \\
\hline Regular alcohol consumption in 2004 & 77 & 0.351 & 0.055 & 0.480 & $0.242-0.460^{*}$ \\
\hline Body mass index in 2014 & 78 & 0.731 & 0.051 & 0.446 & $0.630-0.831$ \\
\hline Body mass index in 2004 & 78 & 0.641 & 0.055 & 0.483 & $0.532-0.750$ \\
\hline Waist-hip ratio in 2014 & 73 & 0.822 & 0.045 & 0.385 & $0.732-0.912$ \\
\hline Waist-hip ratio in 2004 & 73 & 0.863 & 0.041 & 0.346 & $0.782-0.944$ \\
\hline Cardiovascular diseases in 2014 & 78 & 0.269 & 0.051 & 0.446 & $0.169-0.370$ \\
\hline Cardiovascular diseases in 2004 & 78 & 0.269 & 0.051 & 0.446 & $0.169-0.370$ \\
\hline Diastolic blood pressure in 2014 & 69 & 0.377 & 0.059 & 0.488 & $0.260-0.494$ \\
\hline Diastolic blood pressure in 2004 & 69 & 0.217 & 0.050 & 0.415 & $0.118-0.317^{*}$ \\
\hline Systolic blood pressure in 2014 & 69 & 0.348 & 0.058 & 0.480 & $0.233-0.463$ \\
\hline Systolic blood pressure in 2004 & 69 & 0.188 & 0.047 & 0.394 & $0.094-0.283$ \\
\hline
\end{tabular}

*5\% level of statistical significance

history in 45 years. As predictors of meaningfulness within SOC we determined health index in 45 years, systolic blood pressure both in 45 and 55 years, triglycerides both in 45 and 55 years, smoking in 45 years, cardiovascular disease both in 45 and 55 years, diabetes in 55 years, tumors both in 45 and 55 years, and diabetes in family history in 45 years. As predictors of total score of SOC we identified health index in both 45 and 55 years, WHR in 55 years, systolic blood pressure in both 45 and 55 years, total cholesterol in both 45 and 55 years, triglycerides in both 45 and 55 years, alcohol consumption in 55 years, cardiovascular disease both in 45 and 55 years, diabetes in 55 years, tumors both in 45 and 55 years, asthma in both 45 and 55 years, and diabetes in family history in 45 years.

Regarding over limit values as direct questions within the questionnaire (Table 5) we detected alcohol consumption in 2004, diastolic blood pressure in 2004, and systolic blood pressure in 2004.

\section{DISCUSSION}

The sample of participants is specific (longitudinal study), but limited by size ( 88 adults). This is a problem affecting the majority of longitudinal studies. The values of most correlations are relatively low, especially correlations between psychological, somato-physiological and biochemical variables. Nonetheless, we succeeded in obtaining a good deal of statistically significant values.

As regards the comparison of non-communicable disease incidence within the Czech population, our sample of participants of the Czech longitudinal study did not differ from this Czech population sample.

There are a lot of further variables involved in successful development, e.g. resilience, hope (34) or social capital. Broadly construed, they play a role in helping young individuals in particular to negotiate their way out of disadvantage. However, social capital appears to subsume a number of discrete dimensions that are differently linked to particular outcomes $(35,36)$.

\section{Conclusion}

Regardless of the above mentioned limitations of the study, our data should allow greater understanding of the relationships and determinants implicated in health, behavioural, psychological and social variables, predictors and outcomes within the adaptive functioning of personality during the life course within the framework of sustainable development (37).

\section{Acknowledgement}

The study was supported by the Czech Science Foundation as the project P407/10/2410 "Longitudinal study of optimal development: the potential of Prague and Brno longitudinal life-span study".

\section{REFERENCES}

1. Demick J, Andreoletti C, editors. Handbook of adult development. New York: Kluwer Academic; 2003.

2. Lemme BH. Development in adulthood. 2nd ed. Boston: Allyn and Bacon; 1999.

3. Settersten RA Jr. Age structuring and the rhythm of the life course. In Mortimer JT, Shanahan MJ, editors. Handbook of the life course. New York: Kluwer Academic; 2003. p. 81-98.

4. Wapner S, Demick J. Adult development: the holistic, developmental, and systems-oriented perspective. In: Demick J, Andreoletti C, editors. Handbook of adult development. New York: Kluwer Academic; 2003. p. 63-84.

5. Elder GH Jr, Kirkpatrick Johnson, M, Crosnoe, R. The emergence and development of life course theory. In: Mortimer JT, Shanahan MJ, editors. Handbook of the life course. New York: Kluwer Academic; 2003. p. 3-19.

6. Giele JZ, Elder GH Jr. Life course research: development of a field. In: Giele JZ, Elder GH Jr, editors. Methods of life course research: qualitative and quantitative approaches. London: Sage Publications; 1998. p. 5-27.

7. Baltes PB, Staudinger UM, Lindenberger U. Life-span theory in developmental psychology. In: Damon W, editor. Handbook of child psychology. Vol. 1. Theoretical models of human development. 5th ed. New York: Wiley; 1998. p. 1029-143.

8. Magnusson D. Holistic interactionism: a perspective for research on personality development. In: Pervin LA, John OP, editors. Handbook of personality: theory and research. 2nd ed. New York: Guilford Press; 1999. p. 219-47.

9. Ford DH, Lerner RM. Developmental systems theory: an integrative approach. Newbury Park (CA): Sage; 1992. 
10. Lerner RM. Concepts and theories of human development. 3rd ed. Mahwah (NJ): L. Erlbaum Associates; 2002.

11. Brandstädter J. Searching for paths to successful development and aging: integrating developmental and action-theoretical perspectives. In: Pulkkinen L, Caspi A, editors. Paths to successful development: personality in the life course. Cambridge : Cambridge University Press; 2002. p. 380-408.

12. Pulkkinen L, Caspi A. Paths to successful development: personality in the life course. Cambridge: Cambridge University Press; 2002.

13. Aldwin CM, Gilmer DF. Health, illness, and optimal aging : biological and psychosocial perspectives. Thousand Oaks: Sage; 2004.

14. Active ageing: a policy framework. Report No: WHO/NMH/NPH/02.8. Geneva: World Health Organization; 2002.

15. Healthy ageing - a challenge for Europe. Stockholm: Swedish National Institute of Public Health; 2006.

16. Allport GW. Pattern and growth in personality. New York: Holt, Rinehart and Winston; 1961.

17. Rogers CR. On becoming a person; a therapist's view of psychotherapy. Boston: Houghton Mifflin; 1961.

18. Aspinwall LG, Staudinger UM, editors. A psychology of human strengths: fundamental questions and future directions for a positive psychology. Washington: APA; 2003.

19. Havlínová M, Břicháček V, Prokopec M, Kodl M, Kožená L, Tomášek L. Psychological aspects of health behavior in adulthood in individuals longitudinally observed from birth to 45 years. Prague: National Institute of Public Health; 2007. (In Czech.)

20. Costa PT Jr, McCrae RR. Revised NEO Personality Inventory (NEO PI$\mathrm{R})$ and the NEO Five-Factor Inventory (NEO-FFI) professional manual. Odessa (FL): Psychological Assessment Resources; 1992.

21. McCrae RR, Costa PT Jr, OstendorfF, Angleitner A, Hrebickova M, Avia $\mathrm{MD}$, et al. Nature over nurture: temperament, personality, and life span development. J Pers Soc Psychol. 2000;78(1):173-86.

22. Rosenberg M. Society and the adolescent self-image. Princeton (NJ): Princeton University Press; 1965.

23. Schwarzer R. Measurement of perceived self-efficacy. Psychometric scales for cross-cultural research. Berlin: Freie Universität Berlin; 1993.

24. Rokeach M. The nature of human values. New York: The Free Press; 1973.

25. Lukas ES. The Logo-test: a test for measuring personal satisfaction and existentional frustration. Vienna: Deuticke; 1986. (In Deutsch.)
26. Antonovsky A. The structure and properties of the Sense of Coherence Scale. Soc Sci Med. 1993;36(6):725-33.

27. Freedman D, Thornton A, Camburn D, Alwin D, Young-DeMarco L. The Life History Calendar: A technique for collecting retrospective data. Sociol Methodol. 1988;18:37-68.

28. Peterson C, Park N, Seligman MEP. Assessment of character strengths. In: Koocher GP, Norcross JC, Hill SS, editors. Psychologists' desk reference. New York: Oxford University Press; 2005. p. 93-8.

29. Peterson C, Park N, Seligman MEP. Greater strengths of character and recovery from illness. J Posit Psychol. 2006;1(1):17-26.

30. Peterson C, Park N, Pole N, D'Andrea W, Seligman MEP. Strengths of character and posttraumatic growth. J Trauma Stress. 2008;21(2):214-7.

31. Baltes PB, Baltes MM, Freund AM, Lang FR. The measure of selection, optimization, and compensation (SOC) by self-report: technical report 1999. Berlin: Max Planck Institute for Human Development; 1999.

32. Dunn LM, Dunn LM, William SKT, Wang JJ. Peabody picture vocabulary test-III. Circle Pines (MN): American Guidance Service; 1997.

33. Dollaghan C, Campbell TF. Nonword repetition and child language impairment. J Speech Lang Hear Res. 1998;41(5):1136-46.

34. Šolcová I, Kebza V. Resilience, hope and growth. In: Bormans, L, editor The world book of hope: the source of happiness, success, and strength. Tielt: Lannoo; 2015. p. 166-9.

35. Furstenberg FF Jr, Hughes ME. Social capital and successful development among at-risk youth. J Marriage Fam. 1995 Aug;57(3):580-92.

36. Vyncke V, De Clercq B, Stevens V, Costongs C, Barbareschi G, Jónsson $\mathrm{SH}$, et al. Does neighbourhood social capital aid in levelling the social gradient in the health and well-being of children and adolescents? A literature review. BMC Public Health. 2013 Jan 23;13:65. doi: 10.1186/14712458-13-65.

37. United Nations. Sustainable Development. Knowledge Platform. Post2015 process [Internet]. New York: United Nations; 2014 [cited 2014 May 12]. Available from: http://sustainabledevelopment.un.org/index. php?menu=1561.

Received December 1, 2014 Accepted in revised form December 13, 2015 